Research Article

\title{
Doctor Recommendation Model Based on Ontology Characteristics and Disease Text Mining Perspective
}

\author{
Chunhua Ju $\mathbb{D}^{1}$ and Shuangzhu Zhang $\mathbb{D}^{2}$ \\ ${ }^{1}$ Business Administration College, Zhejiang Gongshang University, Hangzhou, China \\ ${ }^{2}$ School of Management Science and Engineering, Zhejiang Gongshang University, Hangzhou, China
}

Correspondence should be addressed to Shuangzhu Zhang; 16010000028@pop.zjgsu.edu.cn

Received 21 May 2021; Accepted 20 July 2021; Published 9 August 2021

Academic Editor: Yue Zhang

Copyright (C) 2021 Chunhua Ju and Shuangzhu Zhang. This is an open access article distributed under the Creative Commons Attribution License, which permits unrestricted use, distribution, and reproduction in any medium, provided the original work is properly cited.

\begin{abstract}
Background. Patients can access medical services such as disease diagnosis online, medical treatment guidance, and medication guidance that are provided by doctors from all over the country at home. Due to the complexity of scenarios applying medical services online and the necessity of professionalism of knowledge, the traditional recommendation methods in the medical field are confronting with problems such as low computational efficiency and poor effectiveness. At the same time, patients consulting online come from all sides, and most of them suffer from nonacute or malignant diseases, and hence, there may be offline medical treatment. Therefore, this paper proposes an online prediagnosis doctor recommendation model by integrating ontology characteristics and disease text. Particularly, this recommendation model takes full consideration of geographical location of patients. Objective. The recommendation model takes the real consultation data from online as the research object, fully testifying its effectiveness. Specifically, this model would make recommendation to patients on department and doctors based on patients' information of symptoms, diagnosis, and geographical location, as well as doctor's specialty and their department. Methods. Utilizing crawler technique, five hospital departments were selected from the online medical service platform. The names of the departments were in accordance with the standardized department names used in real hospitals (e.g., endocrinology, dermatology, gynemetrics, pediatrics, and neurology). As a result, a dataset consisting of 20000 consultation questions by patients was built. Through the application of Python and MySQL algorithms, replacing semantic dictionary retrieval or word frequency statistics, word vectors were utilized to measure similarity between patients' prediagnosis and doctors' specialty, forming a recommendation framework on medical departments or doctors based on the above-obtained sentence similarity measurement and providing recommendation advices on intentional departments and doctors. Results. In the online medical field, compared with the traditional recommendation method, the model proposed in the paper is of higher recommendation accuracy and feasibility in terms of department and doctor recommendation effectiveness. Conclusions. The proposed online prediagnosis doctor recommendation model integrates ontology characteristics and disease text mining. The model gives a relatively more accurate recommendation advice based on ontology characteristics such as patients' description texts and doctors' specialties. Furthermore, the model also gives full consideration on patients' location factors. As a result, the proposed online prediagnosis doctor recommendation model would improve patients' online consultation experience and offline treatment convenience, enriching the value of online prediagnosis data.
\end{abstract}

\section{Introduction}

As the emphasis of medical care gradually shifts from disease to patient, the role of patients' participation in online health improvement is becoming more prominent. The health service in the world is not only different in terms of regions but also varying in terms of online health services $[1,2]$. Spe- cifically, there exist phenomenon such as information asymmetry between doctors and patients and unequal distribution of medical resources geographically [3]. Therefore, patients registering doctors online and intelligent department recommendation have also become one of the important topics of medical informatization. According to a report released in 2019 by the Big Data Research Institute, the scale of users 
in China's medical and health market was about 800 million by the end of 2018 [4]. With a large number of doctors and patients interacting online, a large amount of real consultation data has been accumulated in the online health community. Therefore, it is of important theoretical and practical value to investigate how to make full use of online data to build models to improve patients' medical treatment experience in terms of increasing the accuracy of patients' medical choice and the effectiveness of department recommendation.

The existing literature has been conducting studies from perspectives of department recommendation and doctor recommendation. The two methods of department recommendation are separately based on expert system and similarity calculation. As for department recommendation based on the expert system, on one hand, through establishment of medical knowledge base with the help from medical experts, the diagnosis process of medical experts is simulated by applying rule-based reasoning engine. As a result, patients' diseases are predicted, so as to achieve the target department recommendation for patients. Moreover, the expert-based department recommendation is built upon fuzzy logic and RBF neural network, effectively improving the recommendation accuracy $[5,6]$. On the other hand, there exist many problems due to the abundant number of reasoning rules, such as low computational efficiency and high maintenance cost of knowledge base. As for department recommendation based on similarity calculation, the current literature uses various methods to measure similarities, such as similarity between patients' symptoms and disease' symptoms [7], TF-IDF sentence-based similarity and TF-IDF algorithm that is based on multiple words $[8,9]$, combination of focus shifting backwards, and professional medical corpus [10]. This similarity-based recommendation would, respectively, calculate the possibility of having disease and descriptive words that may correspond with certain symptoms, realizing the goal of department recommendation to patients. Research of recommendation on doctor is mainly based on the content and collaborative filtering recommendation algorithm, focusing on user keywords, browsing history, evaluation, and other data $[11,12]$. The user collaborative filtering algorithm assumes that one user and other user group who share similar interest would have same product preference [13-15]. Among them, user collaborative filtering algorithm integrating projects mainly solves the problem of information overload through filtering attribute collaboratively [16]. Moreover, the application of customized relational network and tags solves the problem of data sparsity in the matrix factorization recommendation model $[17,18]$, and the collaborative filtering recommendation method integrates contextual perception, project similarity, and user behavior, giving recommendation results from perspectives of patients' contexts, projects, and user participation [19-21]. In addition, scholars also conducted modeling research on doctor recommendation, disease diagnosis, and medical examination [22, 23] from the perspectives of semantic characteristics of medical resources [24], user information types [25], user ratings, and comment portraits [26], as well as Bayesian algorithm [27].

The recommendation algorithms in the traditional medical field mainly have the following three problems. First, in terms of department recommendation, the algorithm based on the expert system causes problems such as explosion of knowledge rule reasoning and high maintenance cost of knowledge base. Furthermore, the algorithm based on similarity may not effectively recognize synonyms, possibly decreasing recommendation accuracy. Second, in terms of doctor recommendation, the user-based collaborative filtering algorithm may cause problems that patients of similar symptoms would not be diagnosed with the same disease, due to complexity and diversity of diseases. What is more, because of the nonnecessary relationship among patients' etiologies, the assumption of the project-based collaborative filtering algorithm that users would choose doctors with the same research field as their previous doctors may hardly be met. Third, although relevant literatures have studied how to reduce data sparsity [28-30], the collaborative filtering recommendation algorithm still cannot completely avoid the performance problems caused by data sparsity.

Based on the above theorization, it can be concluded that the existing recommendation algorithms cannot fully meet requirements with regard to recommendation in the context of the Internet medical field. Patients can access medical services provided by doctors in the online health community all over the country online without going out, including disease diagnosis, medical treatment guidance, and medication guidance. Meanwhile, patients consulting online come from far and near and may involve situations of offline medical treatment, making it necessary to take into account the factor of patients' location. Therefore, this paper proposes an online prediagnosis doctor recommendation model that integrates ontology characteristics and disease text mining, improving both the effectiveness of doctor recommendation within the environment of online medical service and the convenience of offline medical treatment for patients.

\section{Research on the Doctor Recommendation Model}

The doctor recommendation model is mainly divided into three steps. Step 1: data preprocessing. Perform word segmentation and stop word removal with regard to patient's input of natural language. Step 2: hospital department recommendation. After screening patients' query data, create the most similar sentence set based on key parts of word vector or the similarity measurement for symptom descriptions, so as to achieve department recommendation. Step 3: doctor recommendation. Use SQL sentence query in the MYSQL database to complete doctor recommendation (Figure 1).

\section{Data Cleaning Process}

There are mainly two aspects of data that are available online. The first aspect of data is patients' online consultation regarding disease symptom. This source of information mainly covers age, gender, symptom description, and other data. The second aspect of data is doctors' information online, including doctors' names, titles, hospitals, departments, and their specialties as shown in Table 1. All data is in structured form, and information such as disease 


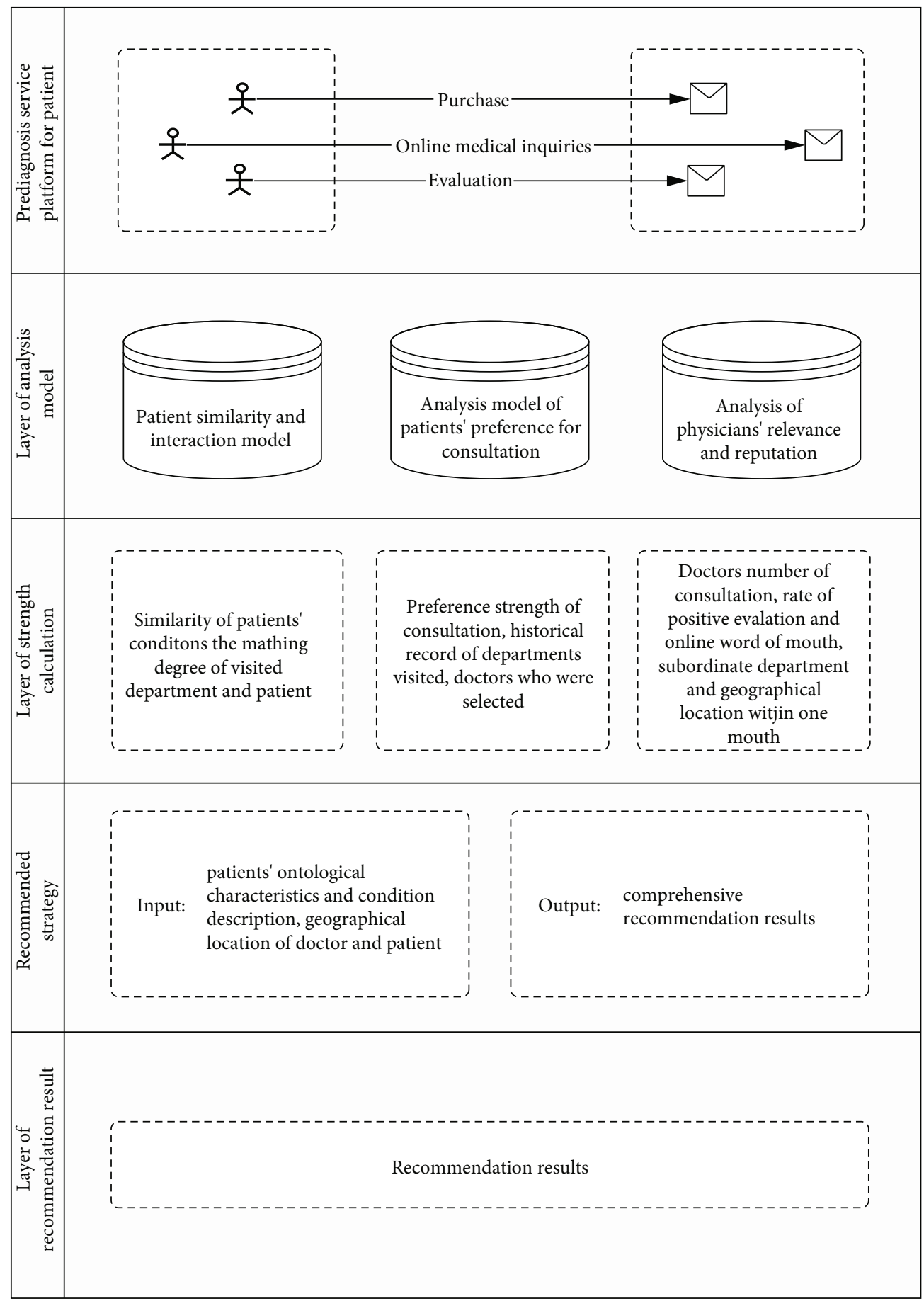

FIgURE 1: Prediagnosis doctor recommendation model integrating ontology characteristics and disease text mining.

description, prediagnosis, and specialties are stored in text form. Then, model will be built after word segmentation and keyword extraction (Figure 2).

\section{Data on Ontology Characteristics of Doctors and Patients}

The doctor-patient demographic data obtained from WeiYi platform are mostly well-organized semistructured textual data. The first step is to transform unstructured text data into structured text data through named entity recognition and information extraction. Organization names, people's names, and location names can be recognized by applying multiple open source Chinese language processing tools [31], such as fudanNLP developed by Fudan University [32], NLPIR word segmentation system developed by Chinese Academy of Sciences [33], and LTP Chinese natural language processing platform of Harbin Institute of Technology [34]. In addition, delete the missing value and duplicated information. And, for the problem of different doctors sharing one same name, use 


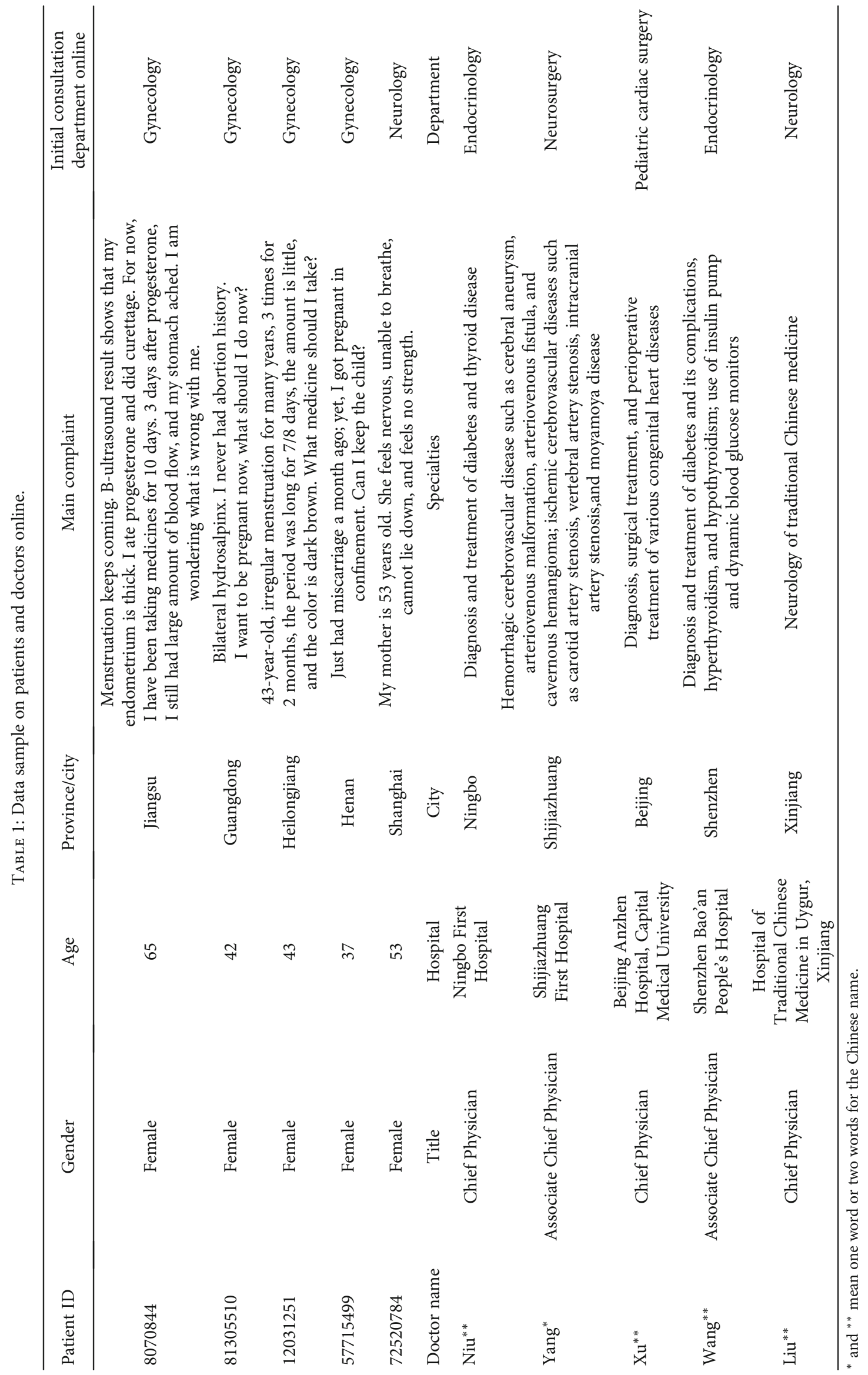




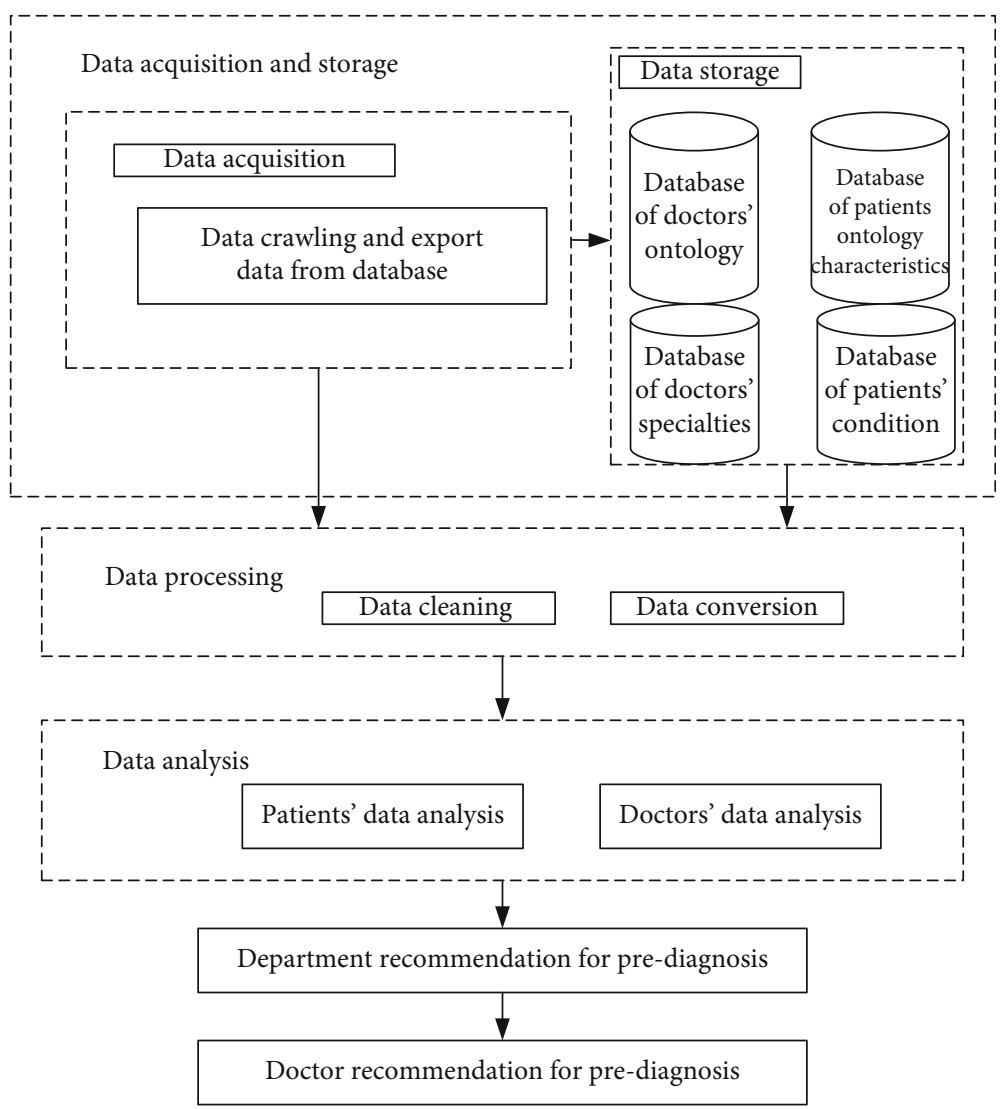

FIgURE 2: Data cleaning process.

fields such as "the hospital to which they belong" and "the department to which they belong" to restrict.

\section{Data on Patients' Condition Description}

Data on patients' online condition description are presented as specific evaluations expressed by patients in natural language. The data in its initial form are fulfilled with problems that the contents are nonstandardized, repetitive, short, and single [35]. The authors marked the text content by part of speech and synonyms and then use human tissue lexicon and human anatomy lexicon to match the word segmentation results so as to extract disease symptoms and keywords of human body parts. As shown in Table 1, the patient's main complaint was that "it was caused by pelvic effusion eight years ago, there was no abortion history and no pregnancy." The common clinical symptoms that the patient did not actually have appeared in the description make it difficult to extract keywords. For example, "no abortion history " was divided into "no" and "abortion history," resulting in the extraction of " abortion history " as the keyword; yet, the patient did not have these symptoms. To deal with situations like the abovementioned, before word segmentation, the authors would divide the description paragraph into short sentences or phrases by punctuation marks, and the stop words should be retained in word segmentation. Then, while extracting keyword, the target words cannot be consid- ered as the real target keywords if they contain negative modifiers such as none, unaccompanied, and no.

\section{Data on Doctors' Specialties}

Data on doctors' specialties are structured textual data and are confronted with problems of synonymous naming and missing data. An example of synonymous naming refers to the problem that doctors in different hospitals have different naming for their fields of expertise. Specifically, synonyms for fields of expertise are specialties, being good at, specializing in, being skilled in, being professional with, medical interest, and research direction. All synonymous naming shall be integrated into the same field. As for the problem of missing data, utilize multiple data source data integration to complete improvement or deletion.

\section{Doctor Recommendation}

7.1. Department Recommendation. For questions input by patients, every keyword for each sentence can be obtained after word segmentation and word stopping removal. Next, the corresponding question set can be obtained by positioning question sentences that are associated with each keyword. The authors divided the question set into sample dataset and test dataset, both containing information of patients' condition description text, online prediagnosis department recommendation, etc. Then, use the word2vec library to train a 


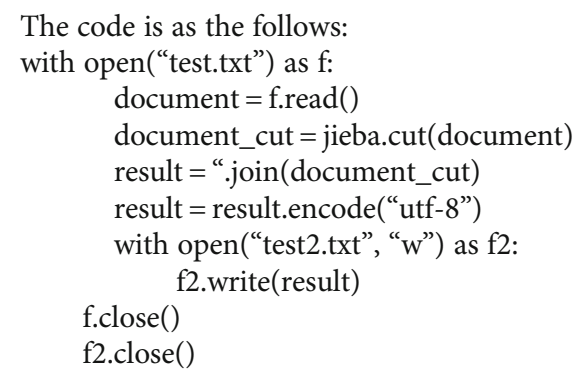

Algorithm 1: This module preprocesses the sample dataset using the following code. The aim is to segment words, remove stop words, and retain key parts or key symptoms with regard to patients' condition description online.

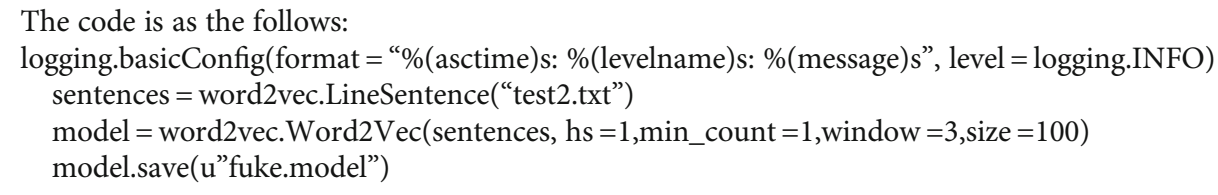

ALgorithm 2: This module used the word2VEC library to train the word vector model of dermatology on sample data such as "dermatology. XLS."

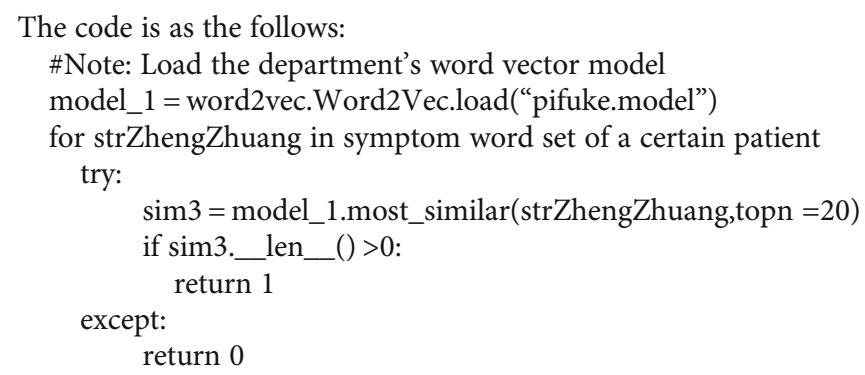

\#Note: Number of matching words/total number of symptom words for a patient = Matching probability probability $=$ Words/WordsCount

Algorithm 3: The module mainly had two goals to achieve. First, preprocess the test data, including word segmentation and stop word removal, and retaining key parts or symptoms for the disease description. Second, compare the word vectors of test data and that of the training results, and the departments with high similarity were recommended to patients.

word vector model on the keywords of the sentences in the sample data set, calculate the similarity between the questions input by the patient in the test data set and the word vector model of the sample data set, and lastly select the most similar questions to the sample data set in the test dataset. Following the rule that higher similarity indicates the same one department, after screening the similarity calculation one by one, the department with the highest similarity would be the final recommendation result.

\section{Doctor Recommendation}

The core significance of the development of online medical and health services is to reshape the medical service process and optimize the allocation of medical resources, so as to meet the medical and health needs of individual consumers.
Due to its mobility, convenience, rapidness, personalization, and interaction, the online medical services have become the main channel for consumers to seek medical help online, having been adopted and utilized by consumers. To some extent, it alleviates the medical pressure and realizes the optimal allocation of medical resources. The patients using online medical service come from all sides, and the majority of them have conventional and chronic diseases, making it sometimes necessary for patients to confirm their diagnosis offline. Therefore, doctor recommendation that takes into account of patients' location information is particularly important to improve patients' convenience of offline medical treatment and to attract more patients to use online medical services. Based on the SQL statements query function in the MYSQL database, matching keywords with doctors' specialties, department, and region information, integrating 
patients' location information, and this paper recommends local doctors that meet the requirements according to patients' region. For instance, a patient's naming Zhang San, living in Zhejiang province, with condition described as thick endometrium, heavy menstrual flow, and stomachache, would be recommended to see a Chief Physician from Department of Gynecology at Zheyi hospital with family name of Wang.

\section{Sentence Similarity}

9.1. Calculation of Similarity Based on Postcontent. After obtaining the unique $d$-dimensional distribution vector representation of the disease description text content, the similarity and distance between each two text contents can be obtained through similarity calculation. The author uses the cosine formula to measure the similarity between two texts and uses the Mahala Nobis distance to calculate the natural language description of the two posts. Assume that two paragraph vectors of natural language description of text content are expressed as $\mathrm{PV}_{a}=(\times 11, \times 12, \cdots, \times 1 d)$ and $\mathrm{PV}_{b}=(\times$ $21, \times 22, \cdots, \times 2 d)$, where $d$ represents two paragraph vectors. The similarity and distance are defined as follows:

$$
\begin{aligned}
\operatorname{sim}(\mathrm{PV} a, \mathrm{PV} b) & =\frac{\mathrm{PV} d \bullet \mathrm{PV} d}{\|\mathrm{PV} d\|^{2} \bullet\|\mathrm{PV} d\|^{2}}, \\
& =\frac{\sum_{i=0}^{i=d} x 1 i x 2 i}{\sqrt{\sum_{i-0}^{i=d} x_{1 i}^{2} \sqrt{\sum_{i-0}^{i=d} x_{2 i}^{2}}}}, \\
\operatorname{dis}(\mathrm{PV} a, \mathrm{PV} b) & =\sqrt{\left(\mathrm{PV} a-\mathrm{PV}_{b}\right)^{T} S^{-1}(\mathrm{PV} a-\mathrm{PV} b)},
\end{aligned}
$$

where $S$ is the covariance matrix of eigenvectors $\mathrm{PV}_{a}$ and $\mathrm{PV}_{b}$.

9.2. TF-IDF Sentence Similarity Based on Co-Occurring Words. This method believes that in two sentences, the more the same vocabulary, the higher the similarity of the two sentences ${ }^{[36]}$. Specifically,

$$
\begin{gathered}
\operatorname{SimScore}(S 1, S 2)=\frac{|S 1 \cap S 2|}{|S 1 \cup S 2|} \sum_{w i \in S 1 \cap S 2} \text { weight }(w i), \\
\operatorname{weight}(w i)=\frac{\operatorname{Num}(w i, k)}{N k} \times \log \left(\frac{N t}{\operatorname{Num}(w i, t)+1}\right) .
\end{gathered}
$$

Among them, $|\cdot|$ is the cardinality of the set, $S_{1}$ and $S_{2}$ are the word sets of the two sentences to be compared, $w_{i}$ represents the symptom word $i$ in the department question and answer sentence, weight $\left(w_{i}\right)$ is the TF-IDF [37] weight, Num $\left(w_{i}, k\right)$ represents the number of sentences in which the symptom word $w_{\mathrm{i}}$ appears in the question and answer sentence set of department $k, N_{k}$ represents the number of all questions and answers in department $k, N_{\mathrm{t}}$ represents the total number of questions and answers in the knowledge base, and Num (wi, $t$ ) represents the total number of ques-
TABLE 2: Summary of the characteristics of the collected data records $(N=20000)$.

\begin{tabular}{lc}
\hline Characteristic & Value, $n(\%)$ \\
\hline Gender & \\
Male & $4540(33.7)$ \\
Female & $15460(77.3)$ \\
Age (years) & \\
25-30 & $1586(7.9)$ \\
$31-45$ & $16800(84.0)$ \\
$46-50$ & $1014(5.1)$ \\
$>55$ & $600(3.0)$ \\
Physician's professional title & \\
Resident physician & $2670(13.35)$ \\
Attending physician & $4330(21.65)$ \\
Associate chief physician & $8040(40.2)$ \\
Chief physician & $4560(22.8)$ \\
Other & $400(2.0)$ \\
Hospital's ranking level & \\
3A & $19400(97.0)$ \\
Other & $600(3.0)$ \\
\hline
\end{tabular}

tions and answers in the knowledge base. The number of sentences in which the symptom word $i$ appears in the question. The TF-IDF sentence similarity calculation method based on co-occurring words belongs to the surface structure analysis method. It simply uses the surface information of the sentence, that is, the word frequency, part of speech, and other information of the words in the sentence to calculate the sentence similarity, without considering synonyms. This results in a decrease in the accuracy of sentence similarity.

9.3. Sentence Similarity Method Based on Word Vector. Word vector sentence similarity is mainly used indepth learning tool word2 $\mathrm{vec}^{[38]}$ to process words into vectors and obtain the semantic similarity of sentence pairs to be compared by calculating the similarity between vectors. The specific formula is as follows:

$$
\begin{gathered}
\underset{w i \in I, w j \in R}{\operatorname{CosSim}}(w i, w j)=\frac{\sum_{i=1}^{n}(x i, y i)}{\sqrt{\sum_{i=1}^{n} x_{i}^{2}} \times \sqrt{\sum_{i=1}^{n} y_{i}^{2}}}, \\
\operatorname{SimScore}(S 1, S 2)=\frac{\sum_{w \in \mathrm{IR}} \beta w M \operatorname{MaxSimValue}(\operatorname{CosSim}(w, \mathrm{IR}))}{\sum_{w \in \mathrm{IR}} \beta w} .
\end{gathered}
$$

Among them, IR $=S_{1} \cup S_{2}, w_{i}$ and $w_{j}$ are the two words to be compared, which represent the words in sentence $S_{1}$ and the words in sentence $S_{2}$, respectively; $n$ represents the dimension of the word vector, and $x_{i}$ and $y_{i}$ represent the word vector of $w_{i}$, and the vector value of the $i$ th dimension of the word vector of $w_{j}$; MaxSimValue $(\operatorname{CosSim}(w, \cdot))$ represents the maximum value of the cosine similarity between the word vector corresponding to word $w$ and the word vector corresponding to all vocabulary of another sentence; 
TABLE 3: Word vector model and keyword examples.

\begin{tabular}{cc}
\hline Word vector-based model & $\begin{array}{c}\text { Keyword set } \\
\text { Headache, nausea, right eye, swelling, stuffy nose, right ear, tinnitus, etc. }\end{array}$ \\
\hline $\begin{array}{c}\text { 1. Migraines, nausea, loss of appetite } \\
\text { Keyword set }\end{array}$ & $\begin{array}{c}\text { Department } \\
\text { Neurology }\end{array}$ \\
3. Headache, dizziness, protrusion of left eye, congestion of eyeball \\
10. Weak right hand, unable to clench a fist, palpitation, unable to breathes \\
\hline
\end{tabular}

parameter $\beta w$ is The TF-IDF weight value of word $w$ in the sentence. The greater the value of SimScore (S1, S2), the greater the similarity between the two sentences and the closer the semantics.

\section{Experiment}

10.1. The Data Set. To analyze the doctor recommendation method proposed in this paper, an experimental study was conducted. The data of five most common departments were crawled from the well-known domestic medical online platform-WeiYi. The names of the departments were in accordance with the standardized department names used in real hospitals (e.g., endocrinology, dermatology, gynemetrics, pediatrics, and neurology). As a result, a dataset with name of T consisting of 20000 patients' preclinical data online were built. To conduct experimentally comparative analysis of various algorithms, two widely used evaluation indexes for the recommendation performance were adopted in this paper, being accuracy rate (being $P$ ) and recall rate (being $R$ ):

$$
P=\frac{\mathrm{TP}}{\mathrm{TP}+\mathrm{FP}} R=\frac{\mathrm{TP}}{\mathrm{TP}+\mathrm{FN}}
$$

10.2. Parameter Setting. In the experiment, the dimension parameter of the word vector was set as 100 . With regard to the calculated similarity results of keyword set that would be used for department recommendation, take the top 5 questions with the highest sentence similarity as the recommended result data (top $N=$ top 5), and the threshold value of keyword set similarity was set as 0.8; that is, when keyword and test set data were used for keyword similarity calculation, the result must exceed 0.8 to be included in the hospital department recommendation set. If there were 2 or more recommended hospital departments, it would be considered as no recommendation, being a special case.

\section{Results and Analysis}

Among the 20000 patients surveyed, 16170 were female (77.3\%). This may be because women are often required to care of family health and other responsibilities in addition to work; also, women tend to pay more attention to health information than men. A total of 16800/20000patients $(84.0 \%)$ were 30 to $45 y$ years of age. Because of old men with limited experiences in consulting physicians and obtaining medicines and children that cannot master online counseling
TABLE 4: Comparison of accuracy and recall rate.

\begin{tabular}{lcc}
\hline Algorithm method & Accuracy rate (\%) & Recall rate (\%) \\
\hline Word vector-based & 74 & 78 \\
Content-based & 63 & 67 \\
Co-occurring word-based & 54 & 56 \\
\hline
\end{tabular}

skills, so, old men and children may not frequently consult physicians on the internet or ask their family members to perform online inquiries. In the 20000 records, 12600 of the physicians $(63.0 \%)$ are chief physicians or associate chief physicians, while19400 hospitals (97.0\%) were ranked 3A (see Table 2).In order to verify the feasibility and effectiveness of the proposed recommendation algorithms for department and doctor, the experiment was conducted to compare them with the content-based recommendation algorithm and user-based collaborative filtering algorithm. First, randomly extract 100 pieces of data from the dataset $T$ based on the hospital department name and then perform word vector training. After the process of word segmentation and stop word removal for data of different departments, the keyword set was obtained, and the word vector model was trained using this keyword set (see Table 3). The word vector model consisted of patients' real consultation questions, and the other words excluding those questions within the group were considered as noise words, representing meaningless words unrelated to patient's consultation. Three different algorithms were all used to measure similarity for keywords to give hospital department recommendation (see results of three algorithms in Table 4).

Seen from Table 4, the proposed similarity recommendation method in this paper that incorporates ontology features and disease text data mining was the best when applied to consultation about selecting appropriate hospital department since the accuracy rate and recall rate were much higher than the other two algorithms. This is because the word vector sentence similarity measurement strategy can better measure the semantic similarity of sentences. For example, for sentence pairs "I went to the hospital to see the dentist and went home, dizzy, heavy head, runny nose" and "When I came back from the dentist, I started to feel Dizziness with symptoms of heavy head and runny nose". If a co-occurring word-based measurement method based on co-occurrence words is used, the similarity value is low, because the sentence pair contains such things as (dizziness, dizziness), (heavy head, sinking head), and (runny nose, runny nose). Synonym pairs such as clear nose) make the content-based 


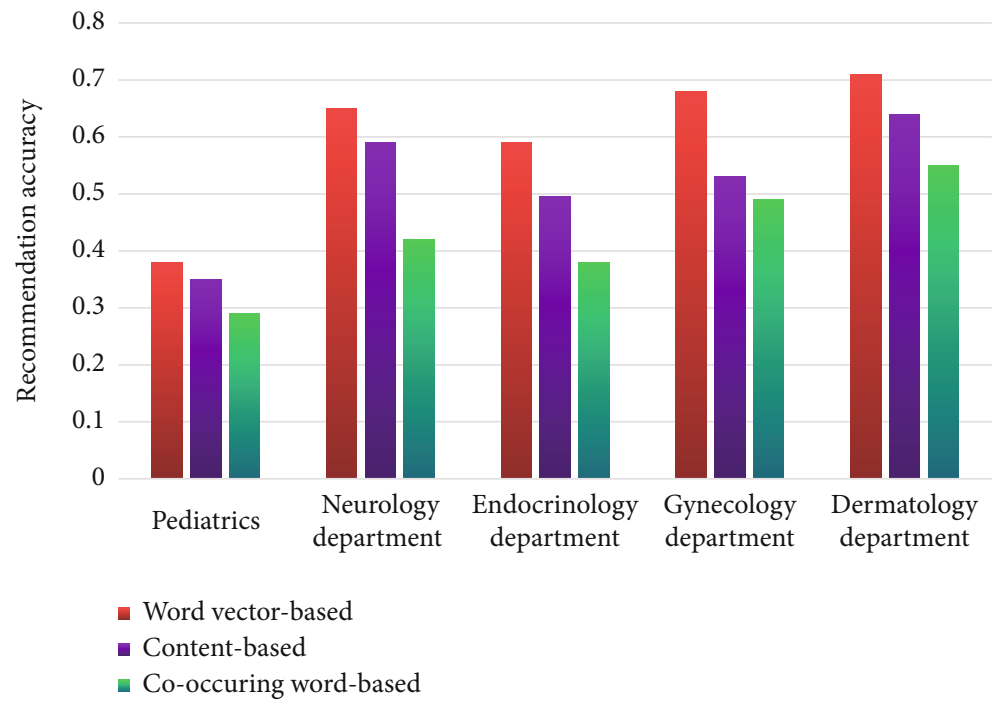

Figure 3: Recommendation accuracy comparison of different departments.

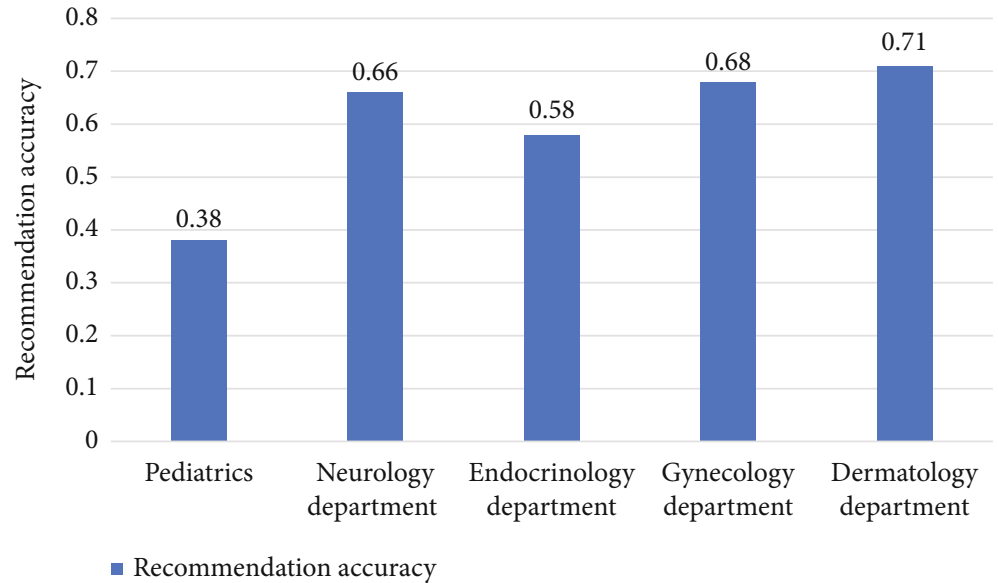

FIGURE 4: Comparison of recommendation rates of various departments.

method relatively good, and the word vector method has the best effect, indicating that it can more accurately capture the underlying semantics of the sentence. On one hand, this is because the method in this paper can measure the similarity of keywords better. For instance, keywords of "headache, palpitation, insomnia" and keywords of "head distension and restlessness" were considered as similar. The results were better than the sentence similarity measurement based on collocates. On the other hand, the proposed method in this paper took fully consideration of factors such as location information of doctors and patients, as well as doctors' expertise field, which would not be the case for the content-based recommendation method that only takes the patient's disease information into account.

Seen from Figures 3 and 4, the recommendation performance of the word vector method was varying for different hospital departments. The recommendation accuracy of pediatric department was below 0.5 , and that of neurology, endocrinology, gynecology, and dermatology departments were all above 0.5 , among which the recommendation accuracy of gynecology was the most improved. With regard to the four departments with relatively higher recommendation accuracy, including neurology, obstetrics, gynecology, and dermatology, what they had in common was that the characteristics of the consultation questions were very typical and obvious. For example, high blood sugar, sudden weight loss, and thirst are typical for endocrinology; red rash, circular rash, redness, swelling, and itching are typical for dermatology; pregnancy and irregular menstruation are typical for gynecology. However, the situation is different for pediatric department in that if information indicating age such as baby, child, and 6 months old is not included in the consultation, it may lead to the systematic recommendation to other departments, reducing the accuracy accordingly.

Finally, The SQL statement query function in the MYSQL database used to integrate the patient's regional factors. According to the patient's region, we use the department and regional keyword matching and recommend the doctors 


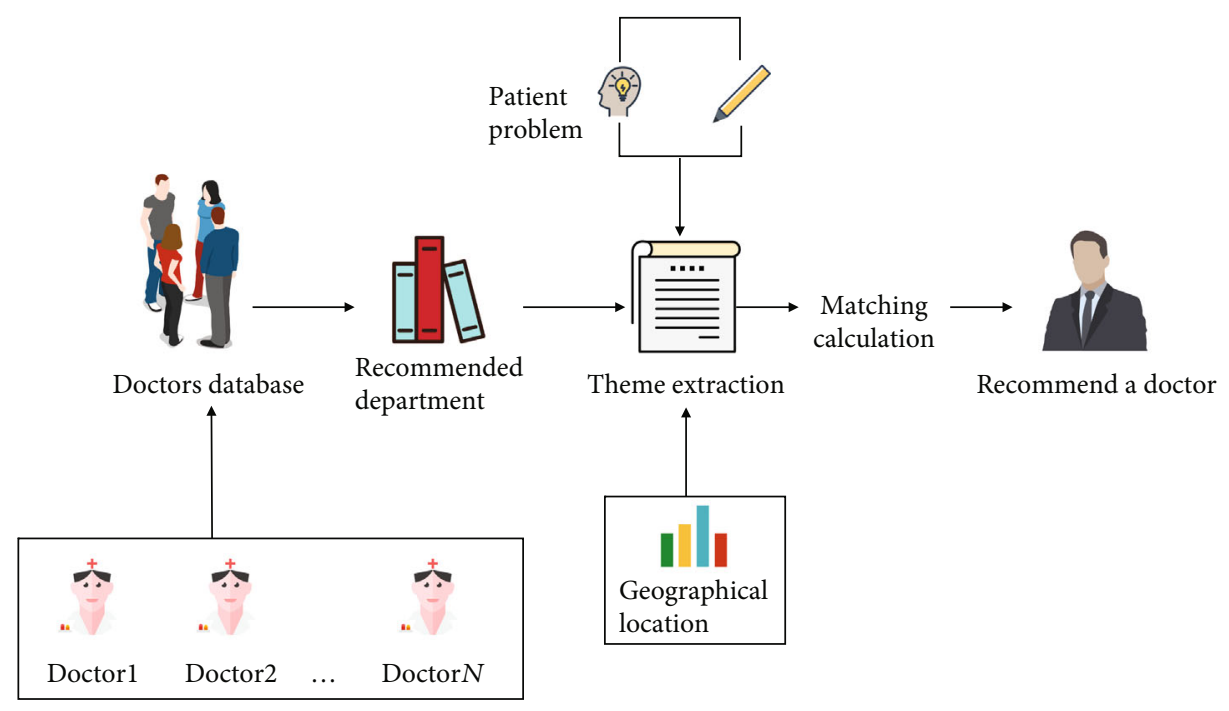

FIgURE 5: Doctor recommendation framework.

in the hospital to patient in the region that meet the needs, such as "Zhang San, from Zhejiang, the condition is described as uterus Thick intima, heavy menstrual flow, and stomachache," and the recommended doctor is "Zhejiang First Hospital-Gynecology-Dr. Wang (Chief Physician)." The process is shown in Figure 5.

\section{Conclusion}

Traditional manual medical guidance is increasingly unable to meet the people's medical needs, registration is difficult, and the problem of not finding a clinic has become increasingly prominent. Aiming at the shortcomings of traditional medical department recommendation research methods and factors such as the necessity for professional medical diagnosis expertise and information asymmetry between doctors and patients makes it impossible for patients to identify the appropriate clinic room or doctors. Once mistakes are made, online consultation time would be wasted, increasing the cost of hospitals and patients when the patient goes offline instead for medical treatment. In this paper, the proposed online prediagnosis doctor recommendation model integrates ontology characteristics and disease text mining. The experimental process uses real data on the Internet medical comprehensive website and is similar to the sentence based on content based, and based on collocate based is compared; the experiment verifies the reliability and effectiveness of the method in this paper. This provides great convenience for patients to seek medical treatment and at the same time reduces medical costs. It gives a relatively more accurate recommendation advice based on ontology characteristics such as patients' description texts and doctors' specialties. As a result, the proposed online prediagnosis doctor recommendation model improves patients' online consultation experience and offline treatment convenience, enriching the value of online prediagnosis data. In addition, the primary real data from the online medical consultation platform were utilized to verify the reliability and effectiveness of the proposed method.

\section{Limitations}

It is not without limitation in this paper. First of all, this study was only carried out based on data from one online medical community, rendering its generalizability a question. Future study may consider collecting data from multiple online medical community platforms to verify the recommendation effect of the proposed algorithm. Second, considering that this study is solely focused on the proposed recommendation model for Chinese patients, similar studies shall be carried out in Western background in the future. Third, because of the complexity of the medical domain knowledge, followup researches shall not only incorporate techniques such as semantic analysis and sentiment analysis to expand the sample into general practice data but also consider introducing users' other behavioral information to introduce the user information behavior factor optimize the target object, for intelligent department recommendation tasks, in addition to controlling data quality and deep learning algorithms such as LSTM shall be applied to improve model accuracy in the future. The intelligent department recommendation task can also be abstracted as a multilabel classification task for texts. Accordingly, multiple department categories can be recommended for patients' questions covering multiple departments, etc. to further improve the accuracy of the proposed recommendation model, expecting to apply it to more online medical consultation platforms.

\section{Data Availability}

The data were collected with help from the administrator of the WeiYi platform. Due to third-party rights, patient privacy, and commercial confidentiality, data is not open source.

\section{Ethical Approval}

The data in this paper is divided into two parts. One part is the information crawled from the platform, such as patient 
comments and doctor profiles. This kind of information is open to the public and everyone can use computer technology to obtain it on the platform. The other part is the patient's age, gender, geographical location, and other information provided by the microdoctor. The WEI-Yi platform is one of the hundreds of online medical platforms in China, with tens of thousands of registered hospitals, registered doctors, and hundreds of thousands of patients using the platform. The platform itself has a sound risk control system, and we have also signed a confidentiality agreement with the platform to define the scope of data use.

\section{Disclosure}

The paper was published in a reduced version at the IEEE 6th International Conference on Big Data Analysis (ICBDA) in 2021.

\section{Conflicts of Interest}

The authors declare that they have no conflicts of interest.

\section{Authors' Contributions}

SZ and CJ refined the topics and methods at the initial stage of paper writing. Then, SZ conducted the statistical analysis and wrote the paper under the guidance of CJ. Both authors reviewed, revised, and approved the final draft.

\section{Acknowledgments}

This project was funded by grants from the National Natural Science Foundation of China: Research on Consumer Credit Value Measurement Integrating Online Social Relationships in eCommerce (71571162). The data were collected with help from the administrator of the WeiYi platform. The data were collected with help from the administrator of the WeiYi platform.

\section{References}

[1] Y. Balarajan, S. Selvaraj, and S. Subramanian, "Health care and equity in India[J]Health care and equity in India," The Lancet, vol. 377, article 9764, pp. 505-515, 2011.

[2] J. M. Goh, G. Gao, and R. Agarwal, "The creation of social value: Can can an online health community reduce ruralurban health disparities?," MIS Quarterly, vol. 40, no. 1, pp. 247-263, 2016.

[3] J. Pan and D. Shallcross, "Geographic distribution of hospital beds throughout China: a county-level econometric analysis," International Journal for Equity in Health, vol. 15, no. 1, p. 179, 2016.

[4] https://data.iimedia.cn/page-category.jsp?nodeid=13210377.

[5] H. Bo, Design and realization of AISCP guiding system built in knowlege base, SUZHOU:SoochowUniversity, 2006.

[6] $\mathrm{H}$. Ru, The design and implementation of the guidance system based on the reasoning algorithm, $\mathrm{H}$. E. FEI, Ed., Anhui University, 2016.

[7] L. Huang, Research on the intelligent medical guide system based on multi-words TF-IDF algorithm, Zhengzhou Univer- sity, Zhengzhou, 2015, https://d.wanfangdata.com.cn/thesis/ D646806.

[8] C. Ju and S. Zhang, "Research on doctor recommendation model for Pre-Diagnosis online based on Big data Mining," in 2021 IEEE 6th International Conference on Big Data Analysis (ICBDA 2021), 2021.

[9] C. Chuan-Peng and W. Zhi-Gang, "A method of sentence similarity computing based on Hownet," Computer Engineering and Science, vol. 34, no. 2, pp. 172-175, 2012.

[10] X. Yifeng, L. Lijun, H. Qingsong, and F. Tiewei, "Research on TF-IDF weight improvement algorithm in intelligent guidance system," Computer Engineering and Applications, vol. 53, no. 4, pp. 238-243, 2017.

[11] X. Hai-Ling, W. Xiao, L. Xiao-Dong, and B.-P. Yan, "Comparison study of internet recommendation system," Journal of Software, vol. 20, no. 2, pp. 350-362, 2009.

[12] C.-G. Huang, J. Yin, J. Wang, Y.-B. Liu, and J.-H. Wang, "Uncertain Neighbors'Collaborative filtering recommendation algorithm," Chinese Journal of Computers, vol. 33, no. 8, pp. 1369-1377, 2010.

[13] Z. Liang and Z. Na, "Improved collaborative filtering algorithm," Computer Systems \& Applications, vol. 25, no. 7, pp. 147-150, 2016.

[14] J. Mingming, Incorporate Topic Model into Collaborative Filtering, Beijing Insititute of Technology, Beijing, 2016.

[15] Y. Wu, T. Rui, and L. Ling, "News recommendation method by fusion of content-based recommendation and collaborative filtering," Journal of Computer Applications, vol. 36, no. 2, pp. 414-418, 2016.

[16] M. López-Nores, Y. Blanco-Fernández, J. J. Pazos-Arias, and A. Gil-Solla, "Property-based collaborative filtering for health-aware recommender systems," Expert Systems with Applications, vol. 39, no. 8, pp. 7451-7457, 2012.

[17] Y. Surong, F. Xiaoqing, and L. Yixing, "Matrix factorization based social recommender model," Journal of Tsinghua University(Science and Technology), vol. 56, no. 7, pp. 793-800, 2016.

[18] F. Bing and N. Xiaoting, "Tag-based matrix factorization recommendation algorithm," Application Research of Computers, vol. 34, no. 4, pp. 1021-1025, 2017.

[19] Z. X. Huang, X. D. Lu, H. L. Duan, and C. Zhao, "Collaboration-based medical knowledge recommendation," Artificial Intelligence in Medicine, vol. 55, no. 1, pp. 13-24, 2012.

[20] J. H. Kim, D. S. Lee, and K. Y. Chung, "Item recommendation based on context-aware model for personalized u-healthcare service," Multimedia Tools and Applications, vol. 71, no. 2, pp. 855-872, 2014.

[21] M. Deshpande and G. Karypis, "Item-based top-N recommendation algorithms," ACM Transactions on Informa- tion Systems, vol. 22, no. 1, pp. 143-177, 2014.

[22] Z. U. O. Chun-tong, Y. U. Ben-gong, and J. I. A. N. G. Shu, "Bayesian networks for knowledge discovery in large medical data Set," Microelectronics \& Computer, vol. 25, no. 6, pp. 113-115, 2008, https://www.wanfangdata.com.cn/details/ detail.do?_type=perio\&id=wdzxyjsj200806030.

[23] B. S. Hu, D. Feng, W. C. Cao, F. LQ, and G. JH, "Mobile intelligent disease diagnosis system based on Bayesian analysis," Journal of Computer Applications, vol. 28, no. 6, pp. 15-17, 2008.

[24] X. Shoukun and W. Weiwei, "Balance recommendation algorthm for medical resources based on semantic," Computer Engineering, vol. 41, no. 9, pp. 74-79, 2015. 
[25] Z. Yan, L. Shiyao, and Z. Can, “An improved recommendation algorithm for mobile health care system," Journal of University of Chinese Academy of Sciences, vol. 34, no. 1, pp. 112-118, 2016.

[26] M. M. Jiang, D. G. Song, L. J. Liao, and F. Zhu, "A Bayesian recommender model for user rating and review profiling," Tsnghua Science and Technology, vol. 20, no. 6, pp. 634-643, 2015.

[27] H. Long-Sheng and Z. Ri-Quan, "0-1 Distributed Bayesian test and application in the medical examination," Application of Statistics and Management, vol. 28, no. 6, pp. 1052-1058, 2009, https://www.wanfangdata.com.cn/details/detail.do?_ type=perio\&id=sltjygl200906013.

[28] M. Xiang-Wu, L. Shu-Dong, Z. Yu-Jie, and H. Xun, "Research on social recommender systems," Journal of Software, vol. 26, no. 6, pp. 1356-1372, 2015.

[29] A. Sheng-sheng, Z. Hai-yan, C. Qing-Kui, and C. Jian, "Probabilistic matrix factorization recommendation algorithm based on social tag and social trust," Journal of Chinese Computer Systems, vol. 37, no. 5, pp. 921-926, 2016, https://www .wanfangdata.com.cn/details/detail.do?_type=perio\&id= xxwxjsjxt201605007.

[30] W. Yang, Z. Yong, L. Zhendong, and Y. Guanci, "Rating prediction algorithm based on semantic similarity and matrix factorization," Journal of Computer Applications, vol. 37, Supplement 1, pp. 287-291, 2017.

[31] F. Xiaoyu, D. Yongxiang, Z. Pengwei, and Z. Xiao, "Study for the construction method of scientist profile with multi source data fusion," Library and Information Service, vol. 62, no. 15, pp. 31-40, 2018.

[32] X. Qiu, Q. Zhang, and X. Huang, "Fudan NLP:a toolkit for Chinese natural language processing," in Proceedings of the meeting of the Association for Computational Linguistics: system demonstrations, pp. 49-54, Sofia: the Association for Computational Linguistics, 2013.

[33] L. Zhou and D. Zhang, "NLPIR: A theoretical framework for applying natural language processing to information retrieval," Journal of the American Society for Information Science and Technology, vol. 54, no. 2, pp. 115-123, 2003.

[34] L. Ting, C. Wanxiang, and L. Zhenghua, "Language technology platform," Journal of Chinese Information Processing, vol. 25, no. 6, pp. 53-62, 2011.

[35] K. Wang, User information extraction and analysis big data environment, Beijing University of Posts and Telecommunications, Beijing, 2018. 